This item was submitted to Loughborough's Research Repository by the author.

Items in Figshare are protected by copyright, with all rights reserved, unless otherwise indicated.

\title{
Observations on the modified Wenker synthesis of aziridines and the development of a biphasic system
}

PLEASE CITE THE PUBLISHED VERSION

http://dx.doi.org/10.1021/jo302615g

PUBLISHER

(C) American Chemical Society

VERSION

AM (Accepted Manuscript)

LICENCE

CC BY-NC-ND 4.0

\section{REPOSITORY RECORD}

Buckley, Benjamin R., Anish P. Patel, and K.G.U. Wijayantha. 2019. "Observations on the Modified Wenker Synthesis of Aziridines and the Development of a Biphasic System". figshare.

https://hdl.handle.net/2134/12027. 
This item was submitted to Loughborough's Institutional Repository (https://dspace.lboro.ac.uk/) by the author and is made available under the following Creative Commons Licence conditions.

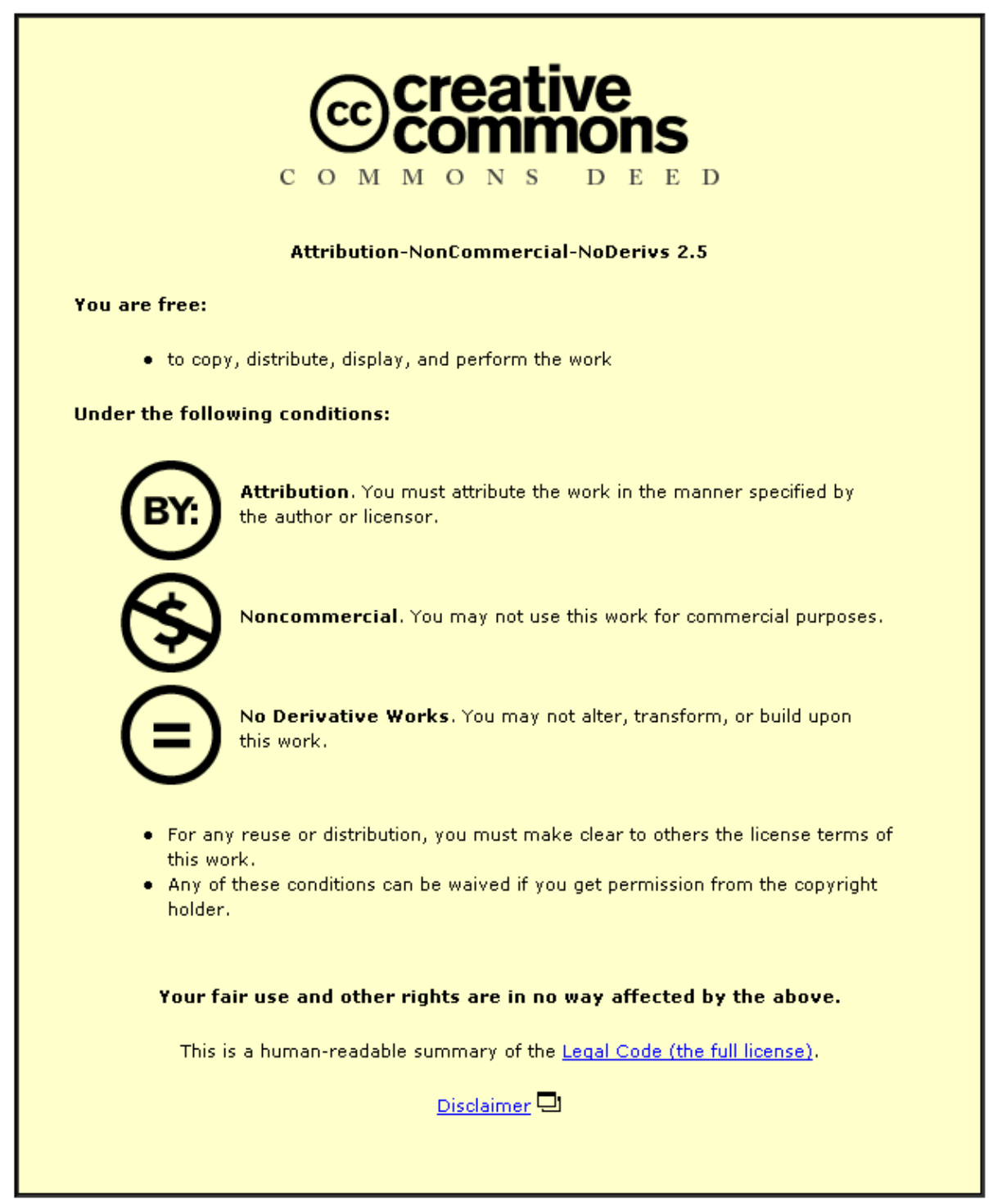

For the full text of this licence, please go to: http://creativecommons.org/licenses/by-nc-nd/2.5/ 


\title{
Observations on the modified Wenker synthesis of aziridines and the development of a biphasic system
}

\author{
Benjamin R. Buckley, * Anish P. Patel and K. G. U. Wijayantha*
}

Department of Chemistry, Loughborough University, Loughborough, Leicestershire, LE11 3TU, UK. Fax: (+)+44 (0)150922 3925,

E-mail: b.r.buckley@Iboro.ac.uk or k.g.u.wijayantha@lboro.ac.uk

\section{TOC Graphic:}

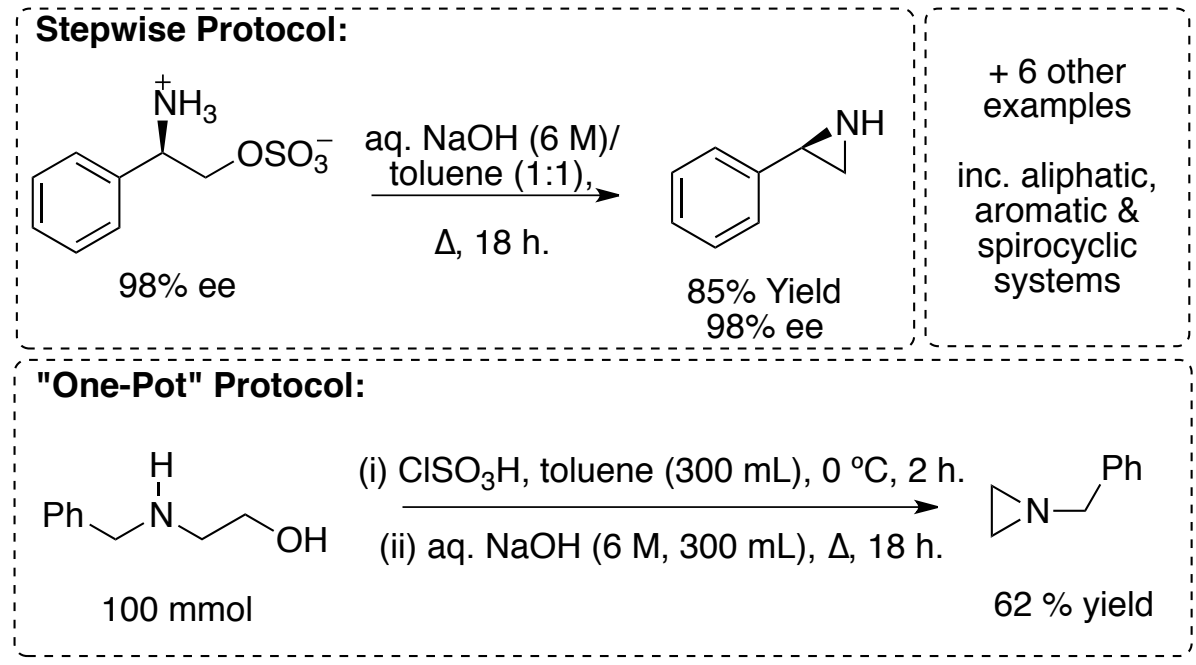

\begin{abstract}
A cheap and reliable process for the modified Wenker cyclization to afford aziridines has been achieved using biphasic conditions for a range of amino alcohol starting materials. A $100 \mathrm{mmol}$ "one-pot" process has also been devised and enantiopurity of the starting amino alcohol is retained in the aziridine product.
\end{abstract}

Aziridines are important synthetic building blocks for the construction of complex nitrogen-containing compounds and are found in several natural products, for example the mitomycins and azicemicins. The construction of the aziridine ring system has been studied intensively over many decades and a host of approaches have been identified, some examples of which are shown in Scheme 1.,2 


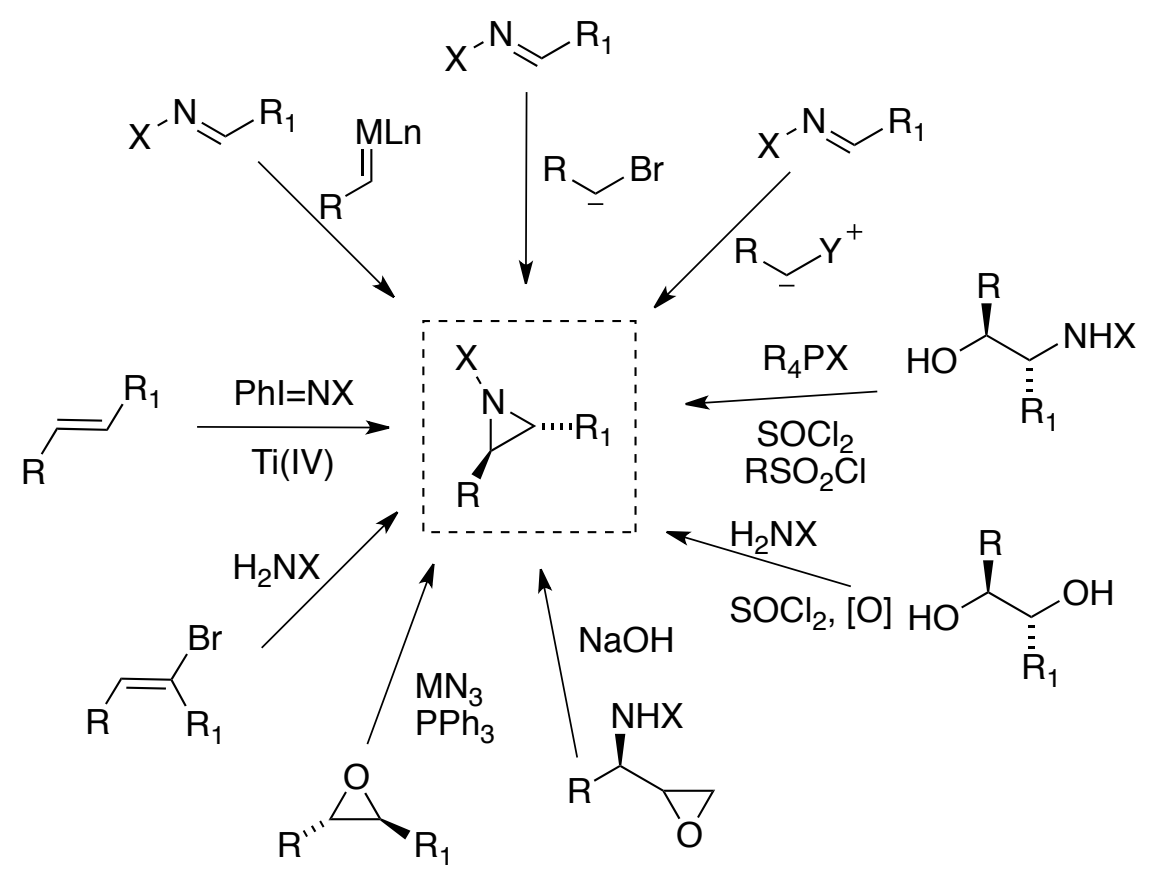

Scheme 1 Common routes towards aziridines

Most approaches require the existence of a protecting group at nitrogen, which is invariably a sulfonate group such as the tosyl group. Approaches towards $\mathrm{N}-\mathrm{H}$ aziridines are much less frequent and perhaps the most well known of these is the Wenker synthesis, in which an amino alcohol is first treated with sulfuric acid and then in a subsequent step with base to ring close to form the desired aziridine. This is an attractive route due to the plethora of starting materials readily available either through synthesis of amino alcohols or by direct purchase from commercial suppliers. However, the harsh reaction conditions employed in the original synthesis (treatment with sulfuric acid and vacuum dehydration) have precluded its use in some circumstances.

For example, $\mathrm{Xu}$ and coworkers have reported some unexpected products that were obtained after treatment of certain amino alcohols under conventional Wenker conditions, in which vicinal amino alcohols in hot sulfuric acid readily undergo elimination of water. $^{3,4}$ In an improved process $\mathrm{Xu}$ and coworkers have converted a range of amino alcohols, under mild conditions, into their corresponding hydrogen sulfate salts with chlorosulfonic acid, the sulfates were then cyclized in good yield with sodium hydroxide or sodium carbonate (Scheme 2 ). ${ }^{5}$

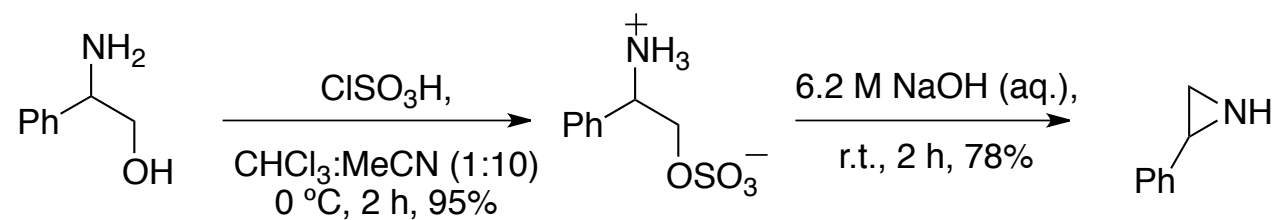


In connection with a number of research programmes in our laboratory we required a mild, reliable, cheap and scalable synthesis of a range of $\mathrm{N}-\mathrm{H}$ aziridines. There are a plethora of reports in this area and we were attracted to the Wenker and related syntheses. ${ }^{6-9}$ Hence we initiated a programme to prepare the aziridines required using the mild conditions reported by $\mathrm{Xu}$ and coworkers above. Synthesis of the hydrogen sulfate adducts 1-6 from amino alcohols was straightforward and proceeded in good to excellent yields, employing chlorosulfonic acid in acetonitrile at $0^{\circ} \mathrm{C}$ (Table 1).

Table 1: Synthesis of the hydrogen sulfate adducts ${ }^{a}$

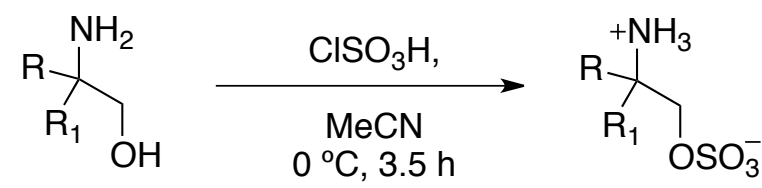

Entry

a General conditions: Amino alcohol (10 mmol), MeCN $(75 \mathrm{~mL}), 0{ }^{\circ} \mathrm{C}$, chlorosulfonic acid (10 mmol), $3.5 \mathrm{~h} . ;{ }^{\mathrm{b}}$ Isolated yield. 
However, in our hands the synthesis of the cyclized aziridines proved troublesome, for example, when using the hydrogen sulfate adduct 1 the sole product produced when employing aqueous $6.2 \mathrm{M} \mathrm{NaOH}$ or saturated aqueous $\mathrm{Na}_{2} \mathrm{CO}_{3}$ at room temperature was phenylglycinol 7. Increasing the reaction temperature to $70^{\circ} \mathrm{C}$ under the $6.2 \mathrm{M}$ $\mathrm{NaOH}$ conditions resulted in a 1:1 mixture of phenylglycinol 7 to aziridine 8, which could be further improved, by heating under reflux, to an 84:16 ratio in favour of the aziridine 8.We found that a screen of the $\mathrm{NaOH}$ concentration only served to increase the ratio of amino alcohol produced (for example, $12 \mathrm{M}$ afforded a 79:21 ratio in favour of the amino alcohol, see Table 2).

Table 2: Attempted synthesis of aziridines under aqueous conditions ${ }^{a}$<smiles>[NH3+]C(CO[Se+3])c1ccccc1</smiles>

1<smiles>NC(CO)c1ccccc1</smiles>

7
8

\begin{tabular}{cccc}
\hline Entry & $\begin{array}{c}\text { (aq.) } \mathrm{NaOH} \\
\text { Concentration }\end{array}$ & $\begin{array}{c}\text { Ratio: }^{\mathrm{b}} \\
\text { Amino Alcohol 7: Aziridine 8 }\end{array}$ \\
\hline 1 & $1 \mathrm{M}$ & 68 & 32 \\
2 & $2 \mathrm{M}$ & 55 & 45 \\
3 & $3 \mathrm{M}$ & 67 & 33 \\
4 & $4 \mathrm{M}$ & 52 & 48 \\
5 & $5 \mathrm{M}$ & 57 & 43 \\
6 & $6 \mathrm{M}$ & 49 & 51 \\
7 & $7 \mathrm{M}$ & 57 & 43 \\
8 & $8 \mathrm{M}$ & 66 & 34 \\
9 & $9 \mathrm{M}$ & 57 & 43 \\
10 & $10 \mathrm{M}$ & 59 & 41 \\
11 & $11 \mathrm{M}$ & 82 & 18 \\
12 & $12 \mathrm{M}$ & 79 & 21 \\
\hline
\end{tabular}

${ }^{a}$ General conditions: $5 \mathrm{mmol}$ substrate, $6.0 \mathrm{M}$ aq. NaOH/co-solvent (1:1), $\Delta 18 \mathrm{~h} ;{ }^{\mathrm{b}}$ Ratio evaluated from the ${ }^{1} \mathrm{H}$ NMR spectrum by integration of the benzylic $\mathrm{C}-\mathrm{H}$ peak, isolated yields were typically $60-80 \%$. 
In an attempt to increase the ratio towards an acceptable and reproducible level of aziridine we opted to add a co-solvent to the reaction, a screen shown in Table 3 shows that the use of toluene under biphasic conditions provides the optimal ratio of aziridine product and an isolated yield of $85 \%$ was consistently observed. 
Table 3: Co-solvent Optimization for the synthesis of aziridines ${ }^{a}$

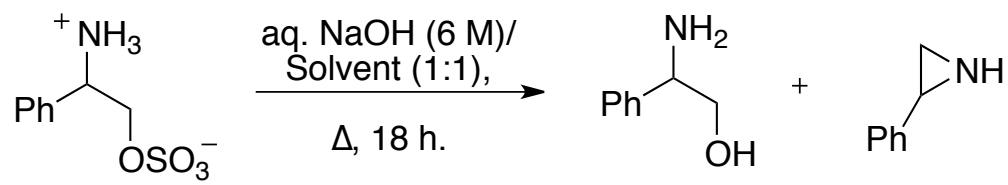

\begin{tabular}{ccccc}
\hline Entry & Co-solvent & \multicolumn{2}{l}{$\begin{array}{l}\text { Ratio: }^{\mathrm{b}} \\
\text { Amino Alcohol 7: Aziridine 8 }\end{array}$} & Yield $^{\mathrm{c}}$ \\
\hline 1 & None & 16 & 84 & $\mathrm{nd}$ \\
2 & Toluene & 5 & 95 & $85 \%$ \\
3 & EtOAc & $<5$ & $<5$ & $\mathrm{nd}^{\mathrm{d}}$ \\
4 & THF & 19 & 81 & $\mathrm{nd}$ \\
5 & i-PrOH & 50 & 50 & $\mathrm{nd}$ \\
6 & MeCN & $<5$ & $<5$ & $\mathrm{nd}^{\mathrm{d}}$ \\
7 & Acetone & $<5$ & $<5$ & $\mathrm{nd}^{\mathrm{d}}$ \\
\hline
\end{tabular}

${ }^{a}$ General conditions: $5 \mathrm{mmol}$ substrate, $6.0 \mathrm{M}$ aq. NaOH/co-solvent (1:1), $\Delta 18 \mathrm{~h}$; ${ }^{\mathrm{b}}$ Ratio evaluated from the ${ }^{1} \mathrm{H}$ NMR spectrum by integration of the benzylic $\mathrm{C}-\mathrm{H}$ peak; ${ }^{\mathrm{c}}$ isolated yield after chromatography; ${ }^{d}$ Complex mixture observed by ${ }^{1} \mathrm{H}$ NMR spectroscopy

Having optimized the conditions for aziridine synthesis from phenyl glycinol hydrogen sulfate we then went on to apply these conditions over a range of substrates for aromatic, aliphatic and spirocyclic aziridine formation (Table 4). Good to excellent yields were obtained, however, we encountered problems with several substrates due to their volatility hence we opted to isolate these as the corresponding tosylate, with yields over the two steps varying from 55 to $65 \%$.

In order to test the robustness of the procedure we carried out the cyclization of the phenyl glycinol salt 1 on a larger scale $(58 \mathrm{mmol})$, gratifyingly we observed a $80 \%$ yield of aziridine. We also carried out the process with enantioenriched starting material and observed complete retention of stereochemical information in the aziridine product (98\% ee, Scheme 3). 


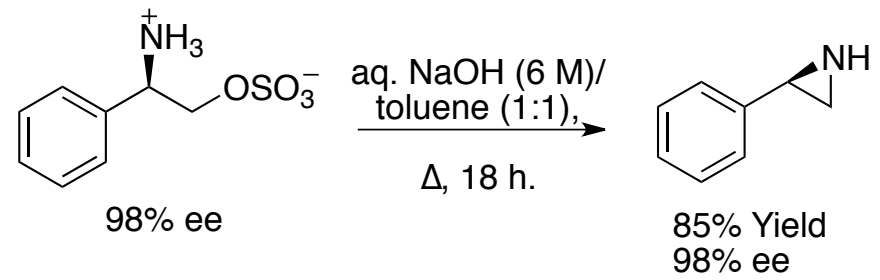

Scheme 3 Retention of stereochemical information after the cyclization process.

Table 4: Aziridine Synthesis ${ }^{a}$

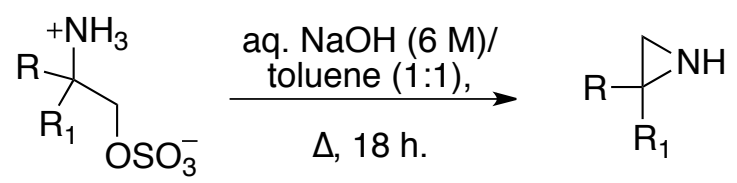

Entry $\begin{gathered}\text { Hydrogen Sulfate } \\ \text { adduct }\end{gathered}$




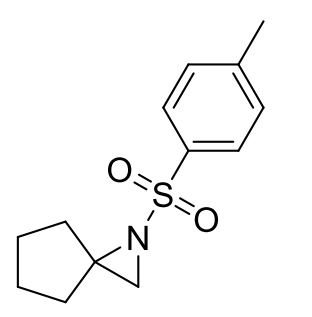

\begin{abstract}
${ }^{a}$ General conditions: Substrate (5 mmol), 6.0 M aq. NaOH/toluene (1:1), $\Delta 18 \mathrm{~h}$; ${ }^{\mathrm{b}}$ Isolated yield after chromatography, yield in parenthesis is from a $58 \mathrm{mmol}$ reaction; ${ }^{\mathrm{c}}$ average yield over three individual runs; ${ }^{d}$ Reaction conditions: (i) Substrate $(5 \mathrm{mmol})$, 6.0 $\mathrm{M}$ aq. $\mathrm{NaOH} /$ toluene (1:1), $\Delta 18 \mathrm{~h}$; (ii) p-tolsulfonyl chloride (5 mmol), $\mathrm{Et}_{3} \mathrm{~N}$ (5 mmol)
\end{abstract} $12 \mathrm{~h}$.

As the initial sulfate salt was prepared in acetonitrile, filtered then re-suspended in toluene before addition of the sodium hydroxide, we were intrigued to find out if the process could be converted to a "one pot" protocol. We therefore dissolved $100 \mathrm{mmol}$ of $\mathrm{N}$-benzylethanolamine in toluene, cooled to $0{ }^{\circ} \mathrm{C}$ and added the chlorosulfonic acid, immediate precipitation of the salt ensued and after $2 \mathrm{~h}$ we directly added sodium hydroxide $(6 \mathrm{M})$ and brought the reaction to reflux. After $18 \mathrm{~h}$ the reaction was worked up in the usual manner and we obtained $62 \%$ yield of the desired aziridine after chromatography.<smiles>OCCNCc1ccccc1</smiles>

$100 \mathrm{mmol}$ (i) $\mathrm{ClSO}_{3} \mathrm{H}$, toluene $(300 \mathrm{~mL}), 0{ }^{\circ} \mathrm{C}, 2 \mathrm{~h}$.

(ii) aq. $\mathrm{NaOH}(6 \mathrm{M}, 300 \mathrm{~mL}), \Delta, 18 \mathrm{~h}$.

$62 \%$ yield

Scheme 4 The "one pot" process.

\title{
Conclusions
}

In conclusion we have developed a reliable and cheap approach to simple aziridines under biphasic conditions. We are now applying this system to a range of aziridines for use in future research areas. 


\section{Experimental Section}

\section{General Experimental}

All infrared thin film spectra were acquired using sodium chloride plates. All ${ }^{1} \mathrm{H}$ and ${ }^{13} \mathrm{C} \mathrm{NMR}$ spectra were measured at 400.13 and $100.62 \mathrm{MHz}$. The solvent used for NMR spectroscopy was $\mathrm{CDCl}_{3}$ (unless stated otherwise) using TMS (tetramethylsilane) as the internal reference. Chemical shifts are given in parts per million (ppm) and $J$ values are given in Hertz $(\mathrm{Hz})$. All mass spectra were recorded a orbitrap HRMS with an ion max source and ESI probe. Melting points are uncorrected. All chromatographic manipulations used silica gel as the adsorbent. Reactions were monitored using thin layer chromatography (TLC) on aluminium backed plates. TLC visualised by UV radiation at a wavelength of $254 \mathrm{~nm}$, or stained by exposure to an ethanolic solution of phosphomolybdic acid (acidified with concentrated sulfuric acid), followed by charring where appropriate. Purification by column chromatography used silica adsorbent. Reaction solvents were obtained commercially dry, and were used without further purification. Enantiomeric excess was determined by chiral HPLC. A Eurocel 01 (manufactured by Knauer, Chiracel OD equivalent) column was used for the determination of enantiomeric excess of the non-racemic styrene aziridine reaction on a HPLC instrument, with the ultraviolet absorption detector set at $254 \mathrm{~nm}$, attached to a data station. 
Representative Procedure for the Formation of the Aminosulfate Salts: Amino alcohol $(10 \mathrm{mmol})$ was dissolved in acetonitrile $(75 \mathrm{~mL})$, the reaction mixture was cooled to $0{ }^{\circ} \mathrm{C}$ followed by drop-wise addition of chlorosulfonic acid $(1.17 \mathrm{~g}, 0.66 \mathrm{~mL}$, $10 \mathrm{mmol}$ ). The resulting heterogeneous solution was stirred constantly at room temperature for $3.5 \mathrm{~h}$. The reaction mixture was then filtered under vacuum, followed by washing with ethyl acetate and diethyl ether. The filter cake was dried in air under vacuum and then transferred to a RBF and placed under high vacuum to afford the aminosulfate salt as a colorless solid.

\section{2-Ammonio-2-phenylethyl sulfate $1:^{10}$}

Colorless solid (1.76 g, 81\%), mp 245-246 ${ }^{\circ} \mathrm{C}$ (lit. ${ }^{10} 247{ }^{\circ} \mathrm{C}$ ); ${ }^{1} \mathrm{H}$ NMR $(400 \mathrm{MHz}$, $\left.\mathrm{CDCl}_{3}\right)$ 4.30-4.32 (m, 2H) 4.65-4.69 (m, 1H) 7.38-7.43 (m, 5H) ppm; ${ }^{13} \mathrm{C}$ NMR (100 $\left.\mathrm{MHz}, \mathrm{CDCl}_{3}\right)$ 54.0, 68.7, 127.2, 129.3, 129.8, 132.4 ppm; IR $\left(\mathrm{CH}_{2} \mathrm{Cl}_{2}\right)$ 998, 1161, 1227, 1252, 1458, 1620, $2943 \mathrm{~cm}^{-1}$; HRMS (ESI) calcd. for $\mathrm{C}_{8} \mathrm{H}_{11} \mathrm{NO}_{4} \mathrm{~S}\left(\mathrm{M}^{+} \mathrm{Na}\right) 240.0306$, found 240.0295 .

\section{2-Ammonio-3-phenylpropyl sulfate 2:11}

Colorless solid $(2.08 \mathrm{~g}, 90 \%), \mathrm{mp} 266-272^{\circ} \mathrm{C}$ (lit. $\left.{ }^{11} 265-270{ }^{\circ} \mathrm{C}\right) ;{ }^{1} \mathrm{H}$ NMR $(400 \mathrm{MHz}$, $\left.\mathrm{CDCl}_{3}, \mathrm{Me}_{4} \mathrm{Si}\right)$ 2.90-3.02 (m, 2H) 3.74-3.80 (m, $\left.1 \mathrm{H}\right), 3.99(\mathrm{dd}, J=5.9,11.4 \mathrm{~Hz}, 1 \mathrm{H})$, $4.16(\mathrm{dd}, J=3.1,11.4 \mathrm{~Hz}, 1 \mathrm{H})$ 7.24-7.36 (m, 5H) ppm; ${ }^{13} \mathrm{C}$ NMR (100 MHz, $\mathrm{CDCl}_{3}$, $\left.\mathrm{Me}_{4} \mathrm{Si}\right) 33.7,51.1,65.6,126.8,128.3,128.6,134.0$ ppm; IR $\left(\mathrm{CH}_{2} \mathrm{Cl}_{2}\right)$ 1008, 1170, 1224, 1247, 1457, 1610, $2992 \mathrm{~cm}^{-1}$; HRMS (ESI) calcd. for $\mathrm{C}_{9} \mathrm{H}_{13} \mathrm{NO}_{4} \mathrm{~S}\left(\mathrm{M}^{+} \mathrm{Na}\right)$ 254.0463, found 254.0451.

\section{2-Ammonio-4-methylpentyl sulfate 3:}

Colorless solid (1.38 g, 70\%) mp $287-288^{\circ} \mathrm{C}$, Anal. Calcd for $\mathrm{C}_{6} \mathrm{H}_{15} \mathrm{NO}_{4} \mathrm{~S}: \mathrm{C}, 36.5 ; \mathrm{H}$, 7.7; N, 7.1; Found: C, 36.3; H, 7.5; N 7.0; ${ }^{1} \mathrm{H}$ NMR (400 MHz, $\left.\mathrm{CDCl}_{3}\right) 0.86$ (d, J $6.6 \mathrm{~Hz}$, $6 \mathrm{H}) 1.42-1.53(\mathrm{~m}, 2 \mathrm{H}), 1.60-1.65(\mathrm{~m}, 1 \mathrm{H}) 3.55-3.61(\mathrm{~m}, 1 \mathrm{H}), 4.02(\mathrm{~d}, J=6.6,11.6 \mathrm{~Hz}$, 1H) $4.20(\mathrm{~d}, J=3.2,11.6 \mathrm{~Hz}, 1 \mathrm{H}), \mathrm{ppm} ;{ }^{13} \mathrm{C} \mathrm{NMR}\left(100 \mathrm{MHz}, \mathrm{CDCl}_{3}\right)$ 21.2, 21.6, 23.7 37.3, 49.2, 67.4 ppm; IR $\left(\mathrm{CH}_{2} \mathrm{Cl}_{2}\right)$ 1004,1167, 1228, 1259, $1608 \mathrm{~cm}^{-1}$; HRMS (ESI) calcd. for $\mathrm{C}_{6} \mathrm{H}_{15} \mathrm{NO}_{4} \mathrm{~S}\left(\mathrm{M}^{+} \mathrm{Na}\right) 220.0619$, found 220.0610.

\section{2-(Benzylammonio)ethyl sulfate $4:^{12}$}


Colorless solid $(1.73 \mathrm{~g}, 75 \%) \mathrm{mp} 245-247^{\circ} \mathrm{C}$ (lit. $\left.{ }^{12} 244-246{ }^{\circ} \mathrm{C}\right) ;{ }^{1} \mathrm{H}$ NMR $(400 \mathrm{MHz}$, $\left.\mathrm{CDCl}_{3}\right)$ 3.36-3.38 (m, 2H) 4.25-4.28 (m, 4H), $7.44(\mathrm{~s}, 5 \mathrm{H}) \mathrm{ppm} ;{ }^{13} \mathrm{C} \mathrm{NMR} \mathrm{(100} \mathrm{MHz,}$ $\left.\mathrm{CDCl}_{3}\right)$ 45.9, 51.0, 63.4, 129.3, 129.7, 129.9, 130.4 ppm; IR $\left(\mathrm{CH}_{2} \mathrm{Cl}_{2}\right)$ 1028, 1192, 1214, 1251, 1457, 1613, $3003 \mathrm{~cm}^{-1}$; HRMS (ESI) calcd. for $\mathrm{C}_{9} \mathrm{H}_{13} \mathrm{NO}_{4} \mathrm{~S}\left(\mathrm{M}^{+} \mathrm{Na}\right) 254.0463$, found 254.0449 .

\section{2-Ammonio-3,3-dimethylbutyl sulfate 5:}

Colorless solid (1.68 g, 97\%) mp 278-280 ${ }^{\circ} \mathrm{C}$; Anal. Calcd for $\mathrm{C}_{6} \mathrm{H}_{15} \mathrm{NO}_{4} \mathrm{~S}$ : C, 36.5; $\mathrm{H}$, 7.7; N, 7.1; Found: C, 36.6; H, 7.5; N 6.9; ${ }^{1} \mathrm{H}$ NMR (400 MHz, $\left.\mathrm{CDCl}_{3}\right) 0.98(\mathrm{~s}, 9 \mathrm{H}), 3.31$ (dd, $J=3.3,8.8 \mathrm{~Hz}, 1 \mathrm{H}) 4.08,(\mathrm{dd}, J=8.8,11.4 \mathrm{~Hz}, 1 \mathrm{H}), 4.30$ (dd, $J=3.3,11.4,1 \mathrm{H}$ ) ppm; ${ }^{13} \mathrm{C}$ NMR (100 MHz, CDCl $)$ 25.3, 31.7, 59.3, 65.8 ppm; IR $\left(\mathrm{CH}_{2} \mathrm{Cl}_{2}\right)$ 1009, 1180, 1219, 1246, $1611 \mathrm{~cm}^{-1}$; HRMS (ESI) calcd. for $\mathrm{C}_{6} \mathrm{H}_{15} \mathrm{NO}_{4} \mathrm{~S}\left(\mathrm{M}^{+} \mathrm{Na}\right) 220.0619$, found 220.0609 .

\section{(1-Ammoniocyclopentyl)methyl sulfate 6:}

Colorless solid (1.42 g, 73\%) mp 258-260 ${ }^{\circ} \mathrm{C}$; Anal. Calcd for $\mathrm{C}_{6} \mathrm{H}_{13} \mathrm{NO}_{4} \mathrm{~S}$ : C, 36.9; $\mathrm{H}$, 6.7; N, 7.2; Found: 36.8; $\mathrm{H}, 6.7$; N $7.1{ }^{1} \mathrm{H}$ NMR $\left(400 \mathrm{MHz}, \mathrm{CDCl}_{3}\right)$ 1.67-1.79 (m, 6H), 1.86-1.93 (m, 2H), $\left.4.04(\mathrm{~m}, 2 \mathrm{H}) ;{ }^{13} \mathrm{C} \mathrm{NMR} \mathrm{(100} \mathrm{MHz,} \mathrm{CDCl}_{3}\right)$ 23.8, 33.4, 63.3, 70.6; IR $\left(\mathrm{CH}_{2} \mathrm{Cl}_{2}\right) 999,1108,1207,1246,1634 \mathrm{~cm}^{-1}$; HRMS (ESI) calcd. for $\mathrm{C}_{6} \mathrm{H}_{13} \mathrm{NO}_{4} \mathrm{~S}(\mathrm{M}+\mathrm{Na})$ 218.0463 , found 218.0451 .

\section{General Procedure for the Cyclization to form Aziridines:}

\section{N-H Aziridines:}

Aminosulfate salt $(5.0 \mathrm{mmol})$, was dissolved in $\mathrm{NaOH}(40 \mathrm{~mL}$, aq. $6 \mathrm{M})$ followed by addition of toluene $(40 \mathrm{~mL})$. The resulting biphasic solution was heated under reflux for $18 \mathrm{~h}$ with constant stirring. On completion the organic phase was extracted with ethyl acetate, dried over $\mathrm{MgSO}_{4}$ and evaporated under reduced pressure, followed by purification by column chromatography on silica gel, affording the aziridine as a colorless oil.

\section{N-Ts Aziridines:}

Aminosulfate salt $(5.0 \mathrm{mmol})$, was dissolved in $\mathrm{NaOH}(40 \mathrm{~mL}$, aq. $6 \mathrm{M})$ followed by addition of toluene $(40 \mathrm{~mL})$. The resulting biphasic solution was heated under reflux for $18 \mathrm{~h}$ with constant stirring. On completion the organic phase was extracted with ethyl 
acetate, dried over $\mathrm{MgSO}_{4}$ and evaporated under reduced pressure, followed by addition of $p$-toluenesulfonyl chloride $(0.95 \mathrm{~g}, 5.0 \mathrm{mmol})$ and triethylamine $(0.51 \mathrm{~g} / 0.70$ $\mathrm{mL}, 5.0 \mathrm{mmol}$ ), the resulting reaction mixture was stirred at room temperature for $12 \mathrm{~h}$. On completion the reaction mixture was washed with water, extracted, dried over $\mathrm{MgSO}_{4}$ and evaporated under reduced pressure followed by purification by column chromatography on silica, affording the aziridine as a colorless solid or oil.

\section{2-Phenylaziridine $8:^{13}$}

Colorless oil $(0.51 \mathrm{~g}, 85 \%),{ }^{1} \mathrm{H}$ NMR $\left(400 \mathrm{MHz}, \mathrm{CDCl}_{3}\right) 1.83(\mathrm{~d}, J=3.2 \mathrm{~Hz}, 1 \mathrm{H}), 2.24(\mathrm{~d}$, $J=6.0 \mathrm{~Hz}, 1 \mathrm{H}), 3.04-3.06(\mathrm{~m}, 1 \mathrm{H}), 7.25-7.36(\mathrm{~m}, 5 \mathrm{H}) \mathrm{ppm} ;{ }^{13} \mathrm{C} \mathrm{NMR}\left(100 \mathrm{MHz}, \mathrm{CDCl}_{3}\right)$ 29.3, 32.1, 125.7, 127.1, 128.5, 140.5 ppm; HRMS (ESI) calcd. For $\mathrm{C}_{8} \mathrm{H}_{9} \mathrm{~N}\left(\mathrm{M}^{+} \mathrm{H}\right)$ 120.0808 , found 120.0807 .

\section{2-Benzylaziridine 9: ${ }^{14}$}

Colorless oil $(0.57 \mathrm{~g}, 85 \%),{ }^{1} \mathrm{H}$ NMR $\left(400 \mathrm{MHz}, \mathrm{CDCl}_{3}\right) 1.46(\mathrm{~d}, J=3.6 \mathrm{~Hz}, 1 \mathrm{H}), 1.83(\mathrm{~d}$, $J=4.7 \mathrm{~Hz}, 1 \mathrm{H}), 2.19-2.25(\mathrm{~m}, 1 \mathrm{H}), 2.66(\mathrm{dd}, J=6.0,14.6 \mathrm{~Hz}, 1 \mathrm{H}), 2.81(\mathrm{dd}, J=6.0$, 14.6 Hz, 1H), 7.21-7.33 (m, 5H) ppm; ${ }^{13} \mathrm{C}$ NMR (100 MHz, $\left.\mathrm{CDCl}_{3}\right) 24.8,31.00,40.0$ 126.4, 128.5, 128.8, 139.2 ppm; HRMS (ESI) calcd. For $\mathrm{C}_{9} \mathrm{H}_{11} \mathrm{~N}\left(\mathrm{M}^{+} \mathrm{H}\right)$ 134.0970, found 134.0980 .

\section{2-iso-Butyl-1-tosylaziridine 10: $:^{15}$}

Colorless oil (0.76 g, 60\%), ${ }^{1} \mathrm{H}$ NMR $\left(400 \mathrm{MHz}, \mathrm{CDCl}_{3}\right) 0.88(\mathrm{dd}, J=2.1,4.7 \mathrm{~Hz}, 6 \mathrm{H})$, 1.28-1.40 (m, 2H), 1.57-1.6 (m, 1H), $2.02(\mathrm{~d}, J=4.7 \mathrm{~Hz}, 1 \mathrm{H}), 2.45(\mathrm{~s}, 3 \mathrm{H}) 2.63(\mathrm{~d}, J=$ $7.1 \mathrm{~Hz}, 1 \mathrm{H}), 2.76-2.82(\mathrm{~m}, 1 \mathrm{H}), 7.34(\mathrm{~d}, J=8.5 \mathrm{~Hz}, 2 \mathrm{H},) \mathrm{ppm} ;{ }^{13} \mathrm{C}$ NMR $(100 \mathrm{MHz}$, $\left.\mathrm{CDCl}_{3}\right)$ 21.7, 21.9, 22.8, 26.8, 34.1, 39.1, 40.4, 128.0, 129.6, 135.3, 144.4 ppm; IR $\left(\mathrm{CH}_{2} \mathrm{Cl}_{2}\right) 2958,1494,1376,1365,1184 \mathrm{~cm}^{-1}$; HRMS (ESI) calcd. For $\mathrm{C}_{13} \mathrm{H}_{19} \mathrm{NO}_{2} \mathrm{~S}$ $\left(\mathrm{M}^{+} \mathrm{Na}\right) 276.1034$, found 276.1021 .

\section{1-Benzylaziridine 11: ${ }^{16}$}

Colorless oil (0.40 g, 60\%), ${ }^{1} \mathrm{H}$ NMR (400 MHz, $\left.\mathrm{CDCl}_{3}\right) 1.28(\mathrm{t}, J=2.4 \mathrm{~Hz}, 2 \mathrm{H}) 1.83$ (t, $J=2.4 \mathrm{~Hz}, 2 \mathrm{H}) 3.39(\mathrm{~s}, 2 \mathrm{H}) 7.24-737(\mathrm{~m}, 5 \mathrm{H}),{ }^{13} \mathrm{C} \mathrm{NMR}\left(100 \mathrm{MHz}, \mathrm{CDCl}_{3}\right)$ 27.6, 65.3, 127.0, 128.0, 128.4, 139.3 ppm; IR $\left(\mathrm{CH}_{2} \mathrm{Cl}_{2}\right)$ 1358, 1496, $3031 \mathrm{~cm}^{-1}$; HRMS (ESI) calcd. For $\mathrm{C}_{9} \mathrm{H}_{11} \mathrm{~N}\left(\mathrm{M}^{+} \mathrm{H}\right)$ 134.0970, found 134.0963 . 


\section{2-tert-Butyl-1-tosylaziridine $12:^{17}$}

Colorless solid (0.82 g, 65\%), mp 58-60 ${ }^{\circ} \mathrm{C}$ (lit. $\left.{ }^{17} 58.5-59.2{ }^{\circ} \mathrm{C}\right) ;{ }^{1} \mathrm{H}$ NMR $(400 \mathrm{MHz}$, $\left.\mathrm{CDCl}_{3}\right) 0.79(\mathrm{~s}, 9 \mathrm{H}), 2.17(\mathrm{~d}, J=4.5 \mathrm{~Hz}, 1 \mathrm{H}), 2.44(\mathrm{~s}, 3 \mathrm{H}), 2.49-2.57(\mathrm{~m}, 2 \mathrm{H}), 7.33(\mathrm{~d}, J$ $=8.4 \mathrm{~Hz}, 2 \mathrm{H}), 7.83(\mathrm{~d}, J=8.4 \mathrm{~Hz}, 2 \mathrm{H}) \mathrm{ppm} ;{ }^{13} \mathrm{C} \operatorname{NMR}\left(100 \mathrm{MHz}, \mathrm{CDCl}_{3}\right)$ 21.6, 26.2, 30.1, 30.3, 48.9, 128.2, 129.6, 135.2, 144.4 ppm; IR $\left(\mathrm{CH}_{2} \mathrm{Cl}_{2}\right)$ 2958, 1598, 1368, 1322, $1185 \mathrm{~cm}^{-1}$; HRMS (ESI) calcd. For $\mathrm{C}_{13} \mathrm{H}_{19} \mathrm{NO}_{2} \mathrm{~S}\left(\mathrm{M}^{+} \mathrm{Na}\right) 276.1034$, found 276.1020.

\section{2-Cyclopentyl-1-tosylaziridine $13:^{18}$}

Colorless solid (0.82 g, 65\%), mp 77-79 ${ }^{\circ} \mathrm{C}$ (lit. $\left.{ }^{18} 82-83^{\circ} \mathrm{C}\right) ;{ }^{1} \mathrm{H}$ NMR $\left(400 \mathrm{MHz}, \mathrm{CDCl}_{3}\right)$ 1.66-1.91 (m, 6H) 2.17-2.27 (m, 2H), $2.43(\mathrm{~s}, 3 \mathrm{H}), 2.55(\mathrm{~s}, 2 \mathrm{H}), 7.32(\mathrm{~d}, J=8.3 \mathrm{~Hz}, 2 \mathrm{H})$, $7.83(\mathrm{~d}, J=8.3 \mathrm{~Hz}, 2 \mathrm{H}) \mathrm{ppm} ;{ }^{13} \mathrm{C}$ NMR (100 MHz, $\left.\mathrm{CDCl}_{3}\right)$ 21.6, 25.6, 32.3, 40.7, 56.7, 127.5, 129.5, 137.5, 143.9 ppm; IR $\left(\mathrm{CH}_{2} \mathrm{Cl}_{2}\right)$ 2960, 1599, 1364, 1319, $1184 \mathrm{~cm}^{-1}$; HRMS (ESI) calcd. For $\mathrm{C}_{13} \mathrm{H}_{17} \mathrm{NO}_{2} \mathrm{~S}\left(\mathrm{M}^{+} \mathrm{Na}\right) 274.0878$, found 274.0866.

\section{The one pot Protocol:}

$N$-benzylethanolamine $(15.2 \mathrm{~g}, 100 \mathrm{mmol})$ was dissolved in toluene $(300 \mathrm{~mL})$, the reaction mixture was cooled to $0{ }^{\circ} \mathrm{C}$ followed by drop-wise addition of chlorosulfonic acid $(11.7 \mathrm{~g}, 6.6 \mathrm{~mL}, 100 \mathrm{mmol})$. The resulting heterogeneous solution was stirred constantly at room temperature for $2 \mathrm{~h}$. $\mathrm{NaOH}(300 \mathrm{~mL}$, aq. $6 \mathrm{M})$ was added and the resulting biphasic solution was heated under reflux for $18 \mathrm{~h}$ with constant stirring. On completion the organic phase was separated and the aqueous phase was extracted with ethyl acetate $(3 \times 50 \mathrm{~mL})$. The organics were combined, dried over $\mathrm{MgSO}_{4}$ and evaporated under reduced pressure to afford a pale yellow oil. Purification by column chromatography on silica gel, afforded the aziridine 11 as a colorless oil $(8.25 \mathrm{~g}, 62 \%)$.

\section{Associated Content}

\section{Supporting Information}

Copies of all ${ }^{1} \mathrm{H}$ and ${ }^{13} \mathrm{C}$ NMR spectra. This material is available free of charge via the Internet at http://pubs.acs.org.

\section{Author Information}

Corresponding Author 
*E-mail: b.r.buckley@lboro.ac.uk or k.g.u.wijayantha@lboro.ac.uk

\section{Notes}

The authors declare no competing financial interest.

\section{Acknowledgements}

B.R.B. and K.G.U.W. would like to thank Research Councils UK for RCUK fellowships and Loughborough University for funding a PhD studentship (to A.P.P.). B.R.B. would also like to thank Eli Lilly Co. for an equipment donation grant.

\section{References}

1. Watson, I. D. G. ; Yu, L.; Yudin, A. K. Acc. Chem. Res. 2006, 39, 194.

2. Ibuka, T. Chem. Soc. Rev. 1998, 27, 145.

3. Zhu, M.; Hu, L. B.; Chen, N.; Du, D.-M.; Xu, J. X. Lett. Org. Chem. 2008, 5, 212.

4. (a) Adams, R.; Cairns, T. L. J. Am. Chem. Soc. 1939, 61, 2464. (b) Campbell, K. N.; Campbell, B. K.; McKenna, J. F.; Chaput, E. P. J. Org. Chem. 1943, 8, 103.

5. Li, X.; Chen, N.; Xu, J. Synthesis 2010, 3423.

6. Wenker, H. J. Am. Chem. Soc. 1935, 57, 2328.

7. Leighton, P. A.; Perkins, W. A.; Renquist, M. L. J. Am. Chem. Soc. 1947, 69, 1540.

8. Kashelikar, D. V.; Fanta, P. E. J. Am. Chem. Soc. 1960, 82, 4927.

9. Brois, S. J. J. Org. Chem. 1962, 27, 3532.

10. Dewey, C. S.; Bafford,R. A. J. Org. Chem. 1965, 30, 491.

11. Kashelikar, D. V.; Fanta, P. E. J. Am. Chem. Soc., 1960, 82, 4930.

12. Tomalia, D. A.; Falk, J. C. J. Het. Chem. 1972 , 9, 891.

13. Mison, P.; Chaabouni, R.; Diab, Y.; Martino, R.; Lopez, A.; Lattes, A.; Wehrli, F. W.; Wirthlin, T. Org. Magn. Reson. 1976, 8, 79.

14. El-Abadelah, M. M.; Sabri, S. S.; Jarrar, A. A.; Zarga, M. H. A. J. Chem. Soc., Perkin Trans. 11979, 2881.

15. Samanta, K.; Panda, G. org. Biomol. Chem. 2011, 9, 7365.

16. Bottini, A. T.; Roberts, J. D. J. Am. Chem. Soc. 1958, 80, 5203.

17. Kawamura, K.; Fukuzawa, H.; Hayashi, M. Org. Lett. 2008, 10, 3509. 